\title{
Chaos and coherence in the conservative three-mode decay interaction
}

\author{
M. Frichembruder, R. Pakter, G. Gerhardt, and F. B. Rizzato \\ Instituto de Física, Universidade Federal do Rio Grande do Sul, Caixa Postal 15051, 91501-970 Porto Alegre, \\ Rio Grande do Sul, Brazil \\ (Received 26 April 2000)
}

\begin{abstract}
In this work we analyze the influence of chaos on the coherence of the mismatched three-wave interaction. Chaos starts to play a decisive role in the problem when adiabatic approximations leading to an integrable model for the system cease to be valid. In regular regimes where the field levels are sufficiently small, there is a characteristic value for the frequency mismatch of the triplet below which coherence and phase locking are dominant. In chaotic regimes, on the other hand, there is no such value and locking behaves in a more complicated way.

PACS number(s): 05.45.-a
\end{abstract}

\section{INTRODUCTION}

The conservative three-wave decay interaction is typically seen as a regular process where a higher intensity mode, let us call it mode 1 , delivers energy to other two modes 2 and 3 with smaller amplitudes [1]. If the time scale for energy exchange among the three waves is much longer than the time scale associated with the respective carrier frequencies $\omega_{1}, \omega_{2}, \omega_{3}$, such that one can actually define quasimonochromatic carrier frequencies, and if these frequencies are perfectly matched with $\omega_{1}=\omega_{2}+\omega_{3}$, then the process is fully librational (the orbits are closed in the appropriate phase space) [2] and energy exchange is maximal. Libration is equivalent to the fact that the relative phase of the three modes remains restricted to very limited variations; one says then that the triplet is phase locked. Two factors, however, inhibit the presence of libration and maximal exchange. One of them is the fact that matching is not always exact. It is well known that several wave systems operate under conditions for which one has a mismatched resonance condition $\omega_{1}-\omega_{2}-\omega_{3}=\delta \neq 0$, and that the presence of the mismatch $\delta$ tends to diminish the effective mode interaction [3]. The number of librational orbits is diminished because states that are originally phase locked for $\delta=0$ unlock when the mismatch grows. The relative phase then starts to display slippage as if the modes were noninteracting, at which point it is said that the corresponding orbits open and become rotational [2]. We show that, in regular cases, most of the initial conditions compatible with the setting provided by decay interaction are of the rotational type when $\delta \sim A^{1 / 2} \equiv \delta_{t h r}$, with $A$ as a measure of the total wave energy.

The other factor affecting the character of the interaction is connected with nonintegrability and chaos. Chaos in wave systems occurs when field intensities are large enough, and generally creates or enhances incoherence by broadening the frequency spectrum of the interacting waves, therefore destroying the time periodicity of regular solutions. One thus has to find out under what conditions chaos is a relevant feature in the system and, when present, how it acts upon coherence. The issue is not trivial since in its classical version $[1,3]$ the conservative three-wave coupling can be shown to be integrable. In other words, if chaos is to be present we need some additional and physically relevant mechanism to break down the integrability of the classical version. The problem was partially investigated in a recent paper [4] by de Oliveira, de Oliveira, and Rizzato (we refer to this paper as OOR henceforth) and we briefly sketch their procedures and conclusions here. OOR investigated the three-mode interaction in the context of the Zakharov equations. These equations are a direct generalization of the nonlinear Schrödinger equation and govern the coupled dynamics of high- and low-frequency modes in several situations, as in fluid plasmas and optical systems [5-7] or, with some adaptations, in the generation of relativistic plasma waves for beat wave accelerator applications [8]. Two high-frequency modes can be described by amplitude equations that are first order in time, but a low-frequency mode is also present for which the governing equation is second order. It is the presence of this second order temporal derivative that converts the system into a nonintegrable one. Now if the total wave energy is sufficiently small, there is a big disparity between the time scales respectively associated with energy exchange and with the carrier frequency of the low-frequency mode. Under this condition adiabatic approximations are valid, the second order equation can be approximated by a first order amplitude equation like the other two, and chaos is absent. In this particular situation what one really gets is the interaction of three quasimonochromatic waves as discussed in the previous paragraph. But as energy increases adiabaticity and integrability cease to be valid. It is at this point, for these high levels of energy, that coherence may be affected by chaos. Here we show that one of the waves develops a large frequency bandwidth that can no longer be approximated by narrow spectral wave packets. The presence of $\delta$ had not been considered in OOR and our purpose here is to investigate the combined action of mismatch and chaos. With the help of the concept of limiting curves, we show that in the presence of intense chaotic activity phase slippage always occurs for all orbits and initial conditions when $\delta>0$, even for arbitrarily small mismatches satisfying $0<\delta \ll \delta_{t h r}$. In other words, the trajectories corresponding to all initial conditions become unlocked here. Now when $\delta \leqslant 0$ we show that slippage is absent even in fully chaotic situations where coherence can hardly be said to exist in the sense that periodicity is lost; in this case we would have a partial synchronism among the phases. If we understand coherent exchange 
as a periodic exchange of large amounts of energy between pump and daughter waves, we can summarize our findings by saying that, when chaos is fully developed, slippage and incoherence coexist for $\delta>0$; slippage is absent for $\delta \leqslant 0$ although incoherence is still there due to the aperiodic character of the dynamics. If chaos is absent, the processes are periodic and coherent, but slippage can be present for a fraction of orbits if $\delta>0$. At some points we refer to chaotic regimes with $\delta>0$ as strongly incoherent because they are aperiodic and display slippage simultaneously.

The paper is organized as follows: in Sec. II we introduce our model and make use of adiabatic approximations to perform an initial investigation; in Sec. III we analyze the full nonintegrable dynamics; and in Sec. IV we conclude the work.

\section{THE MODEL AND ITS ADIABATIC APPROXIMATION}

We start from the Zakharov equations describing the interaction of high-frequency electron plasma waves (denoted by the $E$ field below) and low-frequency ion waves (denoted by $n)$ :

$$
\begin{gathered}
i \partial_{t} E+\partial_{x}^{2} E=n E, \\
\partial_{t}^{2} n-\partial_{x}^{2} n=\partial_{x}^{2}|E|^{2} ;
\end{gathered}
$$

$\partial_{\{x, t\}} \equiv \partial / \partial\{x, t\}$. Equation (1) is an amplitude equation which no longer displays high-frequency features, but Eq. (2) is full and provides nonintegrable ingredients.

Then we restrict the analysis to a three-mode truncation, writing

$$
\begin{gathered}
E=a_{1}(t) e^{i k_{1} x}+a_{2}(t) e^{i k_{2} x}, \\
n=a_{3}(t) e^{i k_{3} x}+a_{3}(t) * e^{-i k_{3} x},
\end{gathered}
$$

where perfect wave-vector matching $k_{1}=k_{2}+k_{3}$ is assumed and ion waves are taken to be real. One obtains, after inserting expansions (3) and (4) into Eqs. (1) and (2),

$$
\begin{gathered}
i d_{t} a_{1}(t)=\omega_{1} a_{1}+a_{3} a_{2}, \\
i d_{t} a_{2}(t)=\omega_{2} a_{2}+a_{3}^{*} a_{1}, \\
d_{t}^{2} a_{3}(t)+\omega_{3}^{2} a_{3}=-k_{3}^{2} a_{1} a_{2}^{*},
\end{gathered}
$$

where $\omega_{1,2} \equiv k_{1,2}^{2}$ and $\omega_{3} \equiv k_{3}$, with $d_{t} \equiv d / d t$. The first two equations above, Eqs. (5) and (6), are first order in time but Eq. (7) is second order.

Truncation up to three spatial modes yields accurate results when one works with infinitely extended systems, and in any case the special content of the theory comes from Eq. (7) whose second derivative may be seen as introducing the possibility of a wide frequency bandwidth that can act on coherence.

When $\left|a_{3}\right| \gg\left|a_{1} a_{2}\right|$, one can solve Eq. (7) in the form $a_{3}(t)=\tilde{a}_{3}(t) e^{-i \omega_{3} t}$ with $\tilde{a}_{3}(t)$ as a slowly varying function of time, $\left|d_{t} \tilde{a}_{3}\right| \ll\left|\omega_{3} \tilde{a}_{3}\right|$. If in addition we write $a_{2}$ $=\sqrt{2 / \omega_{3}} \tilde{a}_{2} e^{-i \omega_{2} t}$ and $a_{1}=\sqrt{2 / \omega_{3}} \tilde{a}_{1} e^{-i\left(\omega_{2}+\omega_{3}\right) t}$, a final approximated set can be written in the form

$$
\begin{gathered}
i d_{t} \tilde{a}_{1}(t)=\delta \tilde{a}_{1}+\tilde{a}_{3} \tilde{a}_{2}, \\
i d_{t} \tilde{a}_{2}(t)=\tilde{a}_{3}^{*} \tilde{a}_{1}, \\
i d_{t} \tilde{a}_{3}(t)=\tilde{a}_{1} \tilde{a}_{2}^{*},
\end{gathered}
$$

with

$$
\delta \equiv \omega_{1}-\omega_{2}-\omega_{3}=k_{1}^{2}-k_{2}^{2}-k_{3} .
$$

The above set is entirely first order and integrable. The crucial point in obtaining this expression is the slow modulation assumption applied on Eq. (7) which renders just an adiabatic approximation valid when nonlinearities are so weak that one can tell apart high and low frequencies. In this case we are saying that the low modulational frequency is much smaller than the "high frequency" given by $\omega_{3}=k_{3}$. Another interesting feature of set $(8)-(10)$ is the mismatch $\delta$. This quantity is absent in OOR because there not only is perfect wave-vector matching $k_{1}=k_{2}+k_{3}$ assumed, but also perfect matching of the carrier frequencies $\omega_{1}=\omega_{2}+\omega_{3}$, which demands $k_{1}=\left(1+k_{3}\right) / 2, k_{2}=\left(1-k_{3}\right) / 2$, and $k_{3}>0$ if one recalls that decay instabilities are subject to the additional ordering constraint $\omega_{1}>\omega_{2}$. In the present paper, as mentioned before, we keep $k_{1}=k_{2}+k_{3}$ still, but now allow for the mismatch according to Eq. (11), which requires

$$
\begin{aligned}
& k_{1}=\frac{1+k_{3}}{2}+\frac{\delta}{2 k_{3}}, \\
& k_{2}=\frac{1-k_{3}}{2}+\frac{\delta}{2 k_{3}} .
\end{aligned}
$$

It is advisable to appreciate at this point the role of mismatches in the theory. To do so we proceed to the integration of our mismatched adiabatic set (8)-(10). The usual techniques of nonlinear wave problems indicate that the dynamics can be entirely extracted from an adiabatic, one-degreeof-freedom Hamiltonian $H_{a}$, given by

$$
H_{a}=2 \sqrt{\tilde{\rho}_{1}\left(\frac{A}{2}-\tilde{\rho}_{1}\right)\left(\frac{B}{2}-\tilde{\rho}_{1}\right)} \cos \psi+\delta \tilde{\rho}_{1} .
$$

Use is made of the notations $\tilde{a}_{j}=\sqrt{\tilde{\rho}_{j}} e^{i \phi_{j}}, j=1,2,3$, and $\psi$ $\equiv \phi_{1}-\phi_{2}-\phi_{3}$, and of the Manley-Rowe conserved quantities $\tilde{\rho}_{1}+\tilde{\rho}_{2}=A / 2, \tilde{\rho}_{1}+\tilde{\rho}_{3}=B / 2$. In the Hamiltonian $H_{a}$ the canonical variables are $\tilde{\rho}_{1}$ and $\psi$ and the respective evolution equations are to be derived from $\dot{\tilde{\rho}}_{1}=-\partial H_{a} / \partial \psi$ and $\dot{\psi}$ $=\partial H_{a} / \partial \tilde{\rho}_{1}$. Given this, one can construct the phase space of the problem as the contour levels of function $H_{a}$ $=H_{a}\left(\tilde{\rho}_{1}, \psi\right)$. For the sake of future convenience, in Fig. 1 we represent the phase space in terms of variables $\psi$ and $\rho_{1}=\left(2 / k_{3}\right) \tilde{\rho}_{1}$. We take $k_{3}=1$ and $A=B=0.1$, with $\delta=0$ in panel (a), $\delta=0.4$ in (b), and $\delta=-0.4$ in (c) (here, $\rho_{1}=A$ represents the situation where all the energy is placed in mode 1 , which then plays the role of pump wave). In addition, analysis is restricted to the range $H_{a}<\delta A / 2$ which will be shown to be the one of physical interest. The comparison shows that in the matched case $\delta=0$, orbits cannot move 
(a)

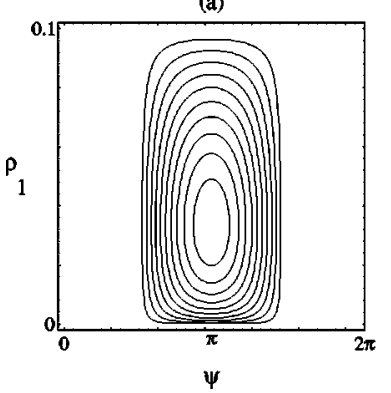

(b)

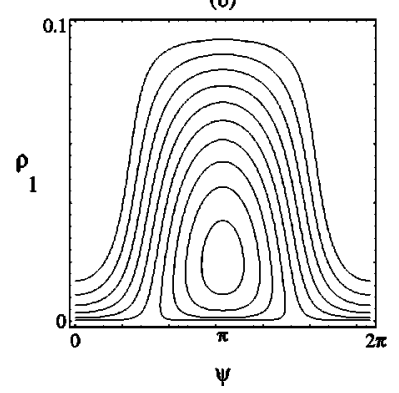

(c)

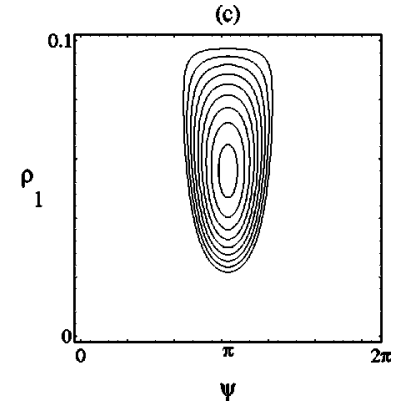

FIG. 1. Contour levels of the adiabatic Hamiltonian $H_{a}$ given in Eq. (14), on the $\left(\psi, \rho_{1}\right)$ phase space; $\delta=0$ in (a), $\delta=0.4$ in (b), and $\delta=-0.4$ in (c). In all cases, $k_{3}=1$ and $A=B=0.1$. over the entire space. The function $\psi=\psi(t)$ will be bounded to vary within the interval $\mathcal{R} \equiv[\pi / 2,3 \pi / 2]$, which in turn implies a reasonable degree of coherence involving the phases. By a reasonable degree of coherence we mean a situation where averages of harmonic functions of $\psi$, like $e^{i \psi}$ to be used later, are not too small. In other words, to have a reasonable degree of coherence we want $\left|\left\langle e^{i \psi}\right\rangle\right| \lesssim 1$ and not $\left|\left\langle e^{i \psi}\right\rangle\right| \ll 1$, with \langle\rangle denoting an average over initial conditions, yet to be better defined. On the other hand, when a positive mismatch is allowed a subset of orbits loses coherence and starts to wander unbounded over the phase space; only orbits sufficiently close to the stable central fixed point remain bounded. Locking is accentuated when $\delta<0$, but then coherent exchange is smaller. In this integrable version of the problem, we note that the size of the incoherent set strongly depends on the magnitude of $\delta$. If $\delta$ is small the incoherent set is also small and should not particularly affect statistical aspects associated with the dynamics. Roughly speaking, untrapped orbits become dominant only when the fixed point drops to a position much below $\tilde{\rho}_{1}=A / 2$ such that the great majority of orbits become untrapped, displaying slippage. The fixed point at $\psi=\pi$ demands $0=\partial H_{a} / \partial \tilde{\rho}_{1}$ from which one gets $\tilde{\rho}_{1} /(A / 2) \sim A^{1 / 2} /(2 \delta)$ if $\tilde{\rho}_{1} \ll A$ and $A$ $=B$ with $k_{3}=1$. This result shows that $\delta$ must exceed a characteristic value

$$
\delta_{i n c} \equiv A^{1 / 2},
$$

which will be referred to as the threshold for untrapping. We finally note that $\delta_{i n c}$ can be large if $A$ is not too small and that $A$ itself can be seen as a measure of the total energy of the system, or, to be more precise, a measure of the maximal pump strength.

The story is remarkably different when chaos is present, however, as the coming analysis will show.

\section{FULL DYNAMICS}

To investigate the full dynamics we use techniques developed in OOR. Let us first show that the dynamics can be derived from a two-degrees-of-freedom Hamiltonian.

We write $a_{1,2}=\left(\sqrt{\rho_{1,2}} / k_{3}\right) e^{i \phi_{1,2}}$ more or less as before, but now use $a_{3}(t)=(1 / \sqrt{2})[u(t)+i v(t)], u, v$ real. This way our governing equations Eqs. (5)-(7) can be entirely derived from the Hamiltonian

$$
\begin{aligned}
H= & \sqrt{2 \rho_{1} \rho_{2}}\left[u \cos \left(\phi_{1}-\phi_{2}\right)-v \sin \left(\phi_{1}-\phi_{2}\right)\right] \\
& +\frac{1}{2}\left[p_{u}^{2}+p_{v}^{2}+k_{3}^{2}\left(u^{2}+v^{2}\right)\right]+\omega_{1} \rho_{1}+\omega_{2} \rho_{2},
\end{aligned}
$$

where $\rho_{1}, \rho_{2}, p_{u}$, and $p_{v}$, are the momenta canonically associated with coordinates $\psi_{1}, \psi_{2}, u$, and $v$, respectively. The governing Hamiltonian (16) is likely to be nonintegrable since no assumption on the time scales of the dynamics is made-i.e., we do not use any modulational approximations to simplify the equations governing the dynamics of mode 3 . A first canonical transformation replaces $\phi_{1} \rightarrow \beta \equiv \phi_{1}-\phi_{2}$ and allows one to rewrite Hamiltonian (16), discarding constant terms, as

$$
\begin{aligned}
H= & \sqrt{2 \rho_{1}\left(A-\rho_{1}\right)}(u \cos \beta-v \sin \beta) \\
& +\frac{1}{2}\left[p_{u}^{2}+p_{v}^{2}+k_{3}^{2}\left(u^{2}+v^{2}\right)\right]+\left(\omega_{3}+\delta\right) \rho_{1},
\end{aligned}
$$

with $A$ as a new momentum satisfying $\rho_{1}+\rho_{2}=A$ and with $\delta$ still given by Eq. (11). One proceeds using polar coordinates $(r, \theta)$ in the form $u=r \sin \theta$ and $v=r \cos \theta$ to write $H$ as

$$
\begin{aligned}
H= & \sqrt{2 \rho_{1}\left(A-\rho_{1}\right)} r \sin (\theta-\beta) \\
& +\frac{1}{2}\left(p_{r}^{2}+k_{3}^{2} r^{2}+\frac{p_{\theta}^{2}}{r^{2}}\right)+\left(\omega_{3}+\delta\right) \rho_{1} .
\end{aligned}
$$

$p_{r}$ and $p_{\theta}$ are momenta conjugate to variables $r$ and $\theta$, respectively. Noticing that the angles appear in the combination $\beta-\theta$, one additional canonical transformation replaces $\beta$ with $\beta \rightarrow \psi \equiv \beta-\theta+\pi / 2$, allowing one to write $p_{\theta}+\rho_{1}$ $=B$ with $B$ as yet another new and conserved momentum. The Hamiltonian therefore takes the final form

$$
\begin{aligned}
H= & \sqrt{2 \rho_{1}\left(A-\rho_{1}\right)} r \cos \psi \\
& +\frac{1}{2}\left(p_{r}^{2}+k_{3}^{2} r^{2}+\frac{\left(B-\rho_{1}\right)^{2}}{r^{2}}\right)+\left(\omega_{3}+\delta\right) \rho_{1} .
\end{aligned}
$$

We set

$$
H=\left(\omega_{3}+\delta\right) A=\left(\omega_{3}+\delta\right) B
$$

to represent decay instabilities. Under this condition the state $\rho_{1}(0)=A$ directly implies $\rho_{2}(0)=0$ and $r(0)=p_{r}(0)=0$, which are indeed the conditions characterizing the initial stage of the decay instability where one has a pump and two daughter waves with vanishing amplitudes. As mentioned earlier, the present work will focus on this particular setting.

\section{A. Limiting curves and Poincaré plots}

Before embarking on the simulations of the full dynamics let us first note that conditions $\omega_{3}=1$ and Eq. (20) along 
(a)

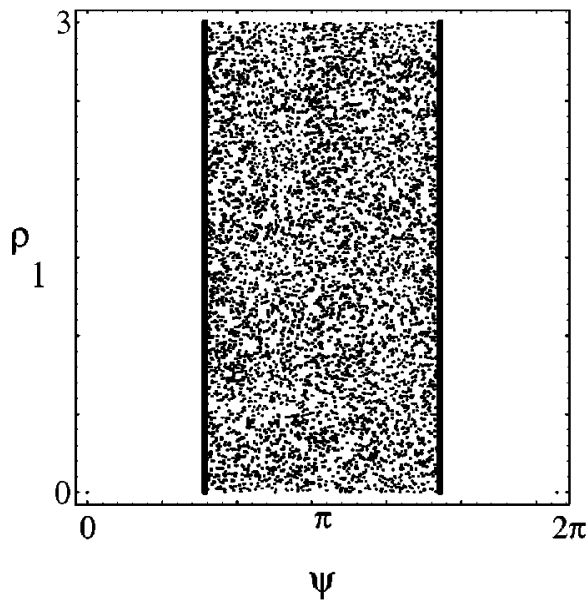

(c)

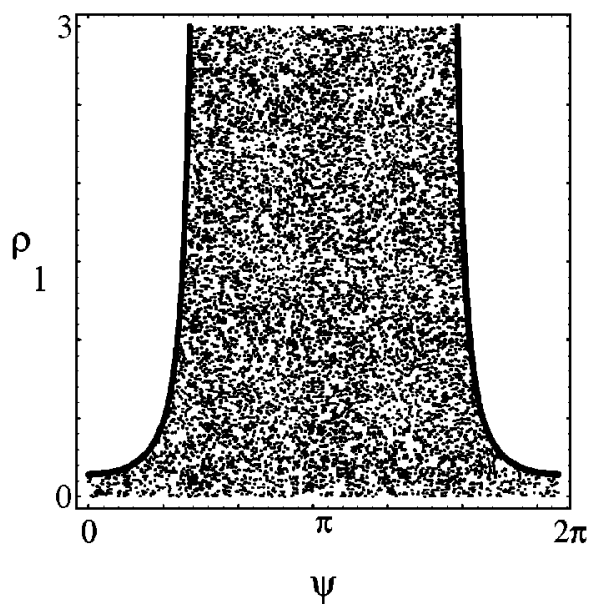

(b)

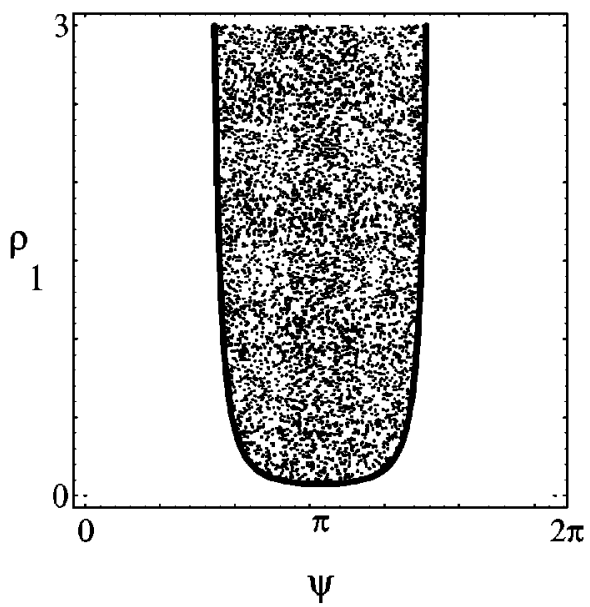

(d)

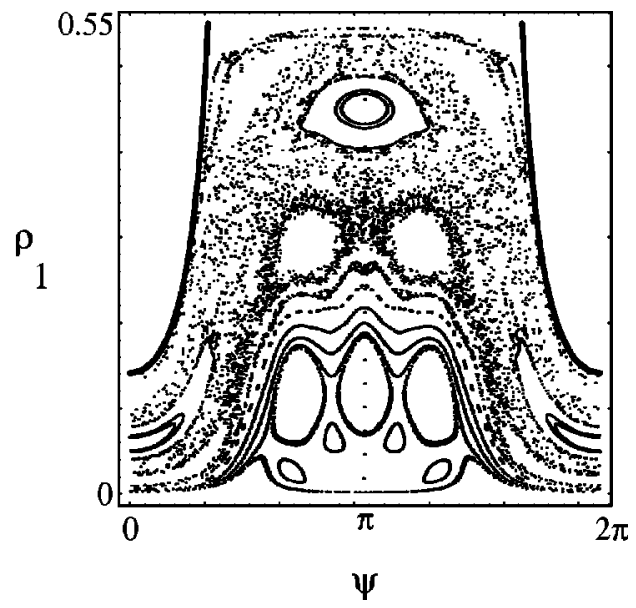

FIG. 2. Limiting curves and Poincaré plots generated by the full Hamiltonian $H$ given in Eq. (19), with $\omega_{3}=1$ and $B=A ; \delta$ $=0$ and $A=3$ in (a), $\delta=-0.4$ and $A=3$ in (b), $\delta=0.5$ and $A=3$ in (c), and $\delta=0.5$ and $A=0.55$ in (d). with the adiabatic approximation $r=\sqrt{A-\rho_{1}}$ coming from the Manley-Rowe conserved quantities arising from Eqs. (8)-(10) allow us to write

$$
2 H_{a}=\delta A-\frac{1}{2} p_{r}^{2},
$$

which explains why we were interested in the regions $H_{a}$ $<\delta A / 2$ to construct the phase plots of Fig. 1 .

But restriction (21) is valid only as long as the dynamics is adiabatic. We wish to see if some constraint of this sort is also present in the full nonintegrable problem. To investigate the issue let us then discuss the existence of limiting curves and their crucial role. Given the constants of motion of the system, limiting curves can be seen as bounding the allowed regions in phase space. Our constants given in relation (20) above yield $p_{r}^{2}$ in the form

$$
\begin{aligned}
\frac{1}{2} p_{r}^{2}= & \left(\omega_{3}+\delta\right)\left(A-\rho_{1}\right)-\sqrt{2 \rho_{1}\left(A-\rho_{1}\right)} r \cos \psi \\
& -\frac{1}{2}\left(k_{3}^{2} r^{2}+\frac{\left(A-\rho_{1}\right)^{2}}{r^{2}}\right) \equiv g(r),
\end{aligned}
$$

which shapes the limiting curve as the one bounding the physical region $g \geqslant 0$, with $g=0$. Now for a given $\rho_{1}$, for instance, a marginally physical value of $\psi$ can be defined as the one where the curve $g=g(r)$ is just tangent to the $g$ $=0$ axis. The marginal value of $\psi$ and the associated $\rho_{1}$ are precisely on the limiting curve. In other words, the curve can be obtained in the form $\rho_{1}=\rho_{1}(\psi)$ if one solves the nonlinear set

$$
g=\frac{d g}{d r}=0 .
$$

In Fig. 2 we represent these curves (solid lines) superposed on Poincare plots which are themselves made by recording the pair $\left(\psi, \rho_{1}\right)$ each time $p_{r}=0$ with $\dot{p}_{r}>0$. We have integrated both the original set (5)-(7) and the equations obtained from the canonically transformed Hamiltonian (19); the simulations display exact agreement, as they should. Various values of $\delta$ and $A$ are considered, along with the usual conditions $\omega_{3}=1$ and $B=A$. When $\delta=0$ the situation is represented as in panel (a) for a high value of $A, A$ $=3$, well above the threshold for chaos. The only allowed region is the region $\mathcal{R}$; phase $\psi$ simply cannot escape elsewhere. This is an interesting result, since even for extremely large fields the orbits are always constrained to move inside a region of relatively limited phase variations. In other words, phase slippage is absent and some sort of partial synchronism of the relative phase $\psi$ persists in the system even for large values of $A$. This partial synchronism remains ac- 
tive for $\delta<0$, but then energy exchange becomes smaller because the accessible phase space is reduced, much like the regular cases analyzed earlier. The dynamics plus limiting curves for $A=3$ and $\delta=-0.4$ are represented in panel (b); there one clearly sees that the allowed region becomes even smaller than in the previous $\delta=0$ case. In all these deeply chaotic cases the phase space seems to be ergodically filled by the orbits. The phase plots suggest that even orbits initially placed in the innermost parts of the allowed regions end up by covering the entire space. One direct tool imported from accelerator physics that can be used to measure ergodicity is the bunching factor $B$ defined in the form

$$
b \equiv\left|\left\langle e^{i \psi}\right\rangle\right| \equiv \frac{1}{N}\left|\sum_{j=1}^{N} e^{i \psi_{j}}\right|
$$

with the averaging summation taken over orbits issuing from $N$ initial conditions [9]. In ergodic cases the average does not depend on the initial conditions and can be evaluated as an integral over allowed regions in phase space,

$$
b_{\text {erg }} \equiv\left|\frac{\int_{\text {allowed }} e^{i \psi} d \psi d \rho_{1}}{\int_{\text {allowed }} d \psi d \rho_{1}}\right| .
$$

When $\delta=0, b_{\text {erg }}$ can easily be evaluated as $b_{\text {erg }}=2 / \pi$ $\approx 0.637$, and, for a sufficiently high value of $A$ so as to produce a good amount of chaos, simulations with $N=100$ initial conditions reveal that the ergodic approximation is good, with $b \rightarrow 0.64$. The practical importance of ergodicity resides in cases with $\delta>0$ as in Fig. 2(c) where we take $\delta=0.5$. Similarly to what happened in the integrable approximation, a small channel is opened near $\rho_{1}=0$, and if ergodicity is present orbits initially placed anywhere inside $\mathcal{R}$ eventually reach the channel and leave $\mathcal{R}$. Synchronism would be broken in view of the fact that the relative phase $\psi(t)$ generated by any initial condition would no longer be constrained to limited variations; note that ergodicity is absent for smaller values of $A$ as seen in Fig. 2(d), where we still take $\delta=0.5$ but now with $A=0.55$. We finally emphasize that the phase behavior in fully chaotic situations is drastically different from the regular approximation. In regular cases incoherence, or slippage, becomes appreciable only when $\delta$ is large enough that $\delta \sim \delta_{t h r}$. For smaller values of $\delta$ one can always find a large fraction of initial conditions placed outside that phase-space region where slippage occurs. But if chaos is present, slippage occurs for any positive, arbitrarily small value of $\delta$, even if these values lie much below the threshold with $\delta \ll \delta_{t h r}$. Now if $\delta$ is very small, $b$ is still close to $2 / \pi$ since the fractional occupation of the channels is small. Therefore one needs a more detailed tool to signal strong incoherence as defined in the Introduction. We investigate the issue next.

\section{B. Phase slippage}

All the discussion of cases with $\delta>0$ can be summarized and made clearer with the help of some additional figures. In what follows, we launch a large number of initial conditions
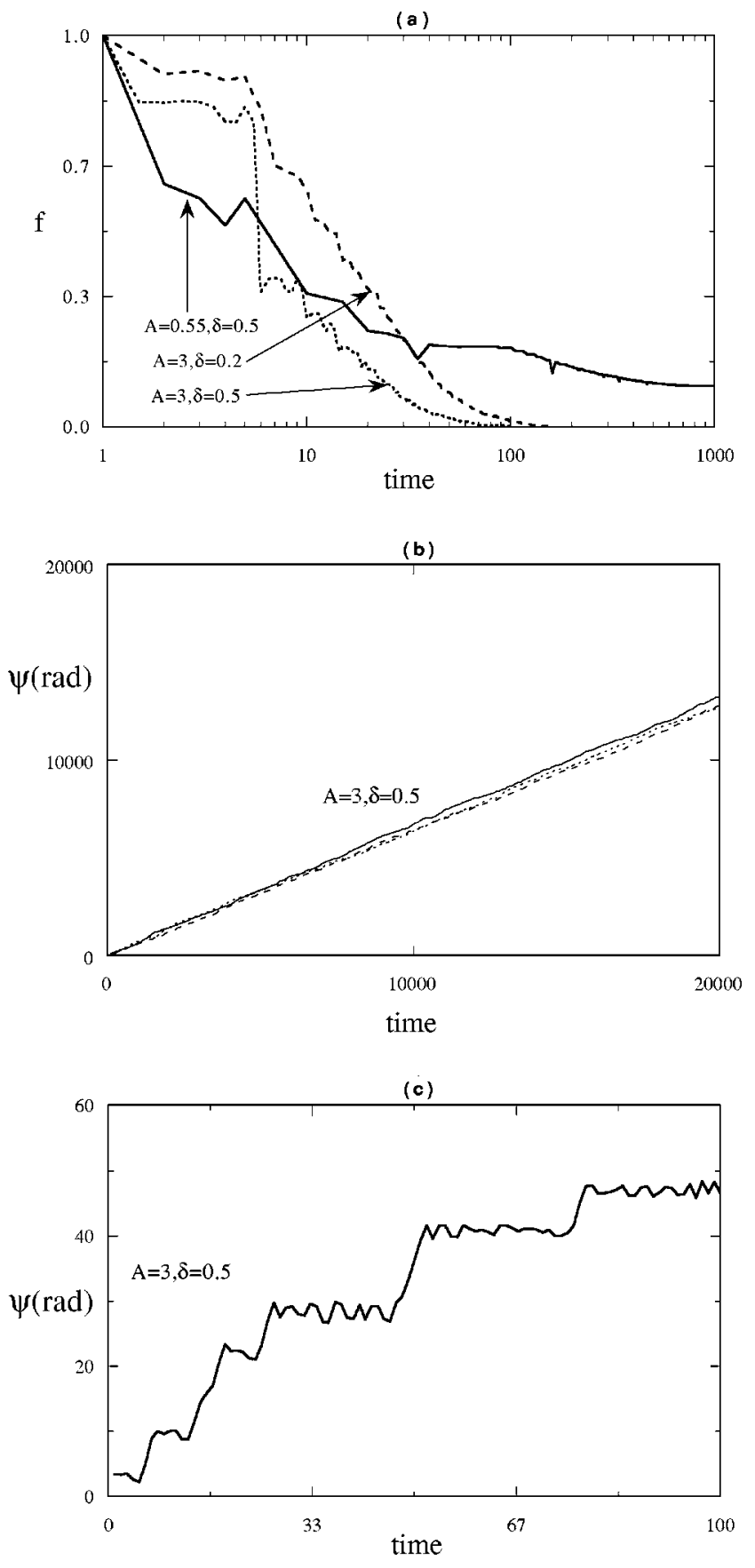

FIG. 3. (a) Fraction $f$ of particles remaining in $\mathcal{R}$ after a time interval $t ; 5000$ initial conditions are uniformly distributed along $\psi=\pi$. (b) $\psi(t)$ versus $t$ for three initial conditions of (a): $\rho_{1}$ $=A / 1.1, A / 3, A / 10$ with $A=3$ and $\delta=0.5 . \omega_{3}=1$ always. (c) $\psi(t)$ versus $t$ for the inital condition $\rho_{1}=A / 3$ of panel (b), on a much finer time scale showing the jumps of the relative phase.

$N=5000$ at $\psi=\pi$ and compute the fraction $f$ of these initial conditions remaining within $\mathcal{R}$ at time $t$.

Figure 3(a) indicates that in regimes of intense chaos with positive $\delta$ as represented by the curves with $A=3$, no trajectory remains trapped in $\mathcal{R}$ and $f \rightarrow 0$ asymptotically. Increasingly large values of $\delta$ only accelerate leakage. For smaller values of $A$ where ergodicity is not complete, as in the case of the curve with $A=0.55$, not all initial conditions abandon $\mathcal{R}$; those initial conditions surrounded by KAM (Kolmogorov-Arnol'd-Moser [10]) curves remain there and $f$ 
no longer vanishes asymptotically [see panel (d) of Fig. 2].

Figure $3(\mathrm{~b})$, with $\psi$ represented not as a modulo $2 \pi$ function, as could perhaps be expected, promptly reveals that in chaotic cases $\psi$ unlocks completely. This panel (b) was made with the version of the integrator where we represent the fields directly in polar coordinates, as in Hamiltonian (19). It seems that unfolding $\psi$ in this way make it easier to realize when we have slippage. In all cases of panel (b) the average phase behavior examined on long time scales mimics the linear time dependence of regular mismatched situations, although the phase velocity $\dot{\psi}$ has not yet been determined in terms of the parameters of the model.

In Fig. 3(c) we run simulations on a much finer time scale than in the previous panel to see that the dynamics is not so smooth as suggested. $\psi$ looks much more erratic-it gets trapped within $\mathcal{R}$, then it jumps away, then it gets trapped in another well, and so on. An average over longer time scales leads to the approximately constant phase velocity of panel (b).

In chaotic cases, if $\delta$ is positive but very small, our results are similar to the case $\delta=0$ in the sense that the orbit spends greater amounts of time within $\mathcal{R}[\bmod 2 \pi]$. But the important point is that, as one increase $\delta$, chaotic orbits start to spend longer and longer time intervals outside $\mathcal{R}[\bmod 2 \pi]$, even when $\delta$ is not large enough to free the majority of orbits of the adiabatic approximation.

One last point we wish to explore is about the effect of $\delta$ on the limiting curves in phase space. Consider Hamiltonian (16). The sum $\omega_{1} \rho_{1}+\omega_{2} \rho_{2}$ on the right-hand side represents the adiabatic normal mode energy of modes 1 and 2 (frequency $\omega$ times wave intensity $\left.|a|^{2} \sim \rho\right)$. The term $(1 / 2)\left[p_{u}^{2}+p_{v}^{2}+k_{3}^{2}\left(u^{2}+v^{2}\right)\right]$ can therefore be seen as representing the energy of mode 3 , which is not adiabatic in our problem, and the first term $\sqrt{2 \rho_{1} \rho_{2}}\left[u \cos \left(\phi_{1}-\phi_{2}\right)-v \sin \left(\phi_{1}\right.\right.$ $\left.\left.-\phi_{2}\right)\right]$ as an interaction potential energy between modes 1 and 2 , and 3 , which we call $V_{\text {int }}$ for short in what follows. Using Manley-Rowe and our initial conditions we can write a final form (19) with $B=A$ and $H=\left(\omega_{3}+\delta\right) A ; V_{\text {int }}$, in particular, takes the form $V_{\text {int }}=\sqrt{2 \rho_{1}\left(A-\rho_{1}\right)} r \cos \psi$. For a given $\rho_{1}$, the lowest energy of mode 3 must correspond to the maximal potential energy $V_{\text {int }}$ and this can give us an idea of the reach of $\psi$. Using the values of $p_{r}$ and $r$ that minimize the energy of mode $3, p_{r}=0$ and $r^{2}=\sqrt{A-\rho_{1}} / \omega_{3}$, we have $H=V_{\text {int }}+\omega_{3} A+\delta \rho_{1}$. If $\delta=0$, as $\rho_{1}$ decreases $V_{\text {int }}$ will be bounded by $H-\omega_{3} A=\omega_{3} A-\omega_{3} A=0$, which implies $\pi / 2 \leqslant \psi \leqslant 3 \pi / 2$. Now, if $\delta>0$, as $\rho_{1}$ decreases $V_{\text {int }}$ must increase to keep $H$ constant, and thus $\cos \psi$ must become positive, opening the locking rectangle; on the other hand, $\delta<0$ shrinks the allowed phase space. $\delta$ can thus be seen almost literally as an excess energy per phonon, relative to the separatrices at $\psi=\pi / 2$ and $3 \pi / 2$. If $\delta>0$ the trajectory clears the separatrix and one has open orbits but if $\delta$ $<0$ one cannot cross the separatrix and only bounded orbits are allowed.

\section{FINAL REMARKS}

In this paper we have analyzed the problem of phase coherence in the conservative nonintegrable three-mode interaction. Using the terminology defined in the Introduction, our main finding is that, while in integrable approximations initial conditions leading to rotational orbits (orbits displaying phase slippage) are more numerous than those leading to librational orbits (orbits displaying locking) as long as the mismatch $\delta$ is sufficiently large to satisfy $\delta>\delta_{t h r} \equiv A^{1 / 2}$, in chaotic regimes phase slippage is always present even when $\delta$ lies much below the threshold with $0<\delta \ll \delta_{t h r}$. In this latter case it does not matter how the initial conditions are prepared; the relative phase of the triplet always breaks loose and any phase synchronism can no longer be observed. Incoherence does occur as higher-order temporal derivatives are introduced in the governing equations broadening the spectral width in frequency space.

\section{ACKNOWLEDGMENTS}

This work was partially supported by Conselho Nacional de Desenvolvimento Científico e Tecnológico (CNPq), Brazil. Some of the numerical computing was performed on the Cray Y-MP2E at the Universidade Federal do Rio Grande do Sul Supercomputing Center. Helpful discussions with P. A. Robinson and P. Drysdale are also acknowledged.
[1] J. Weiland and H. Wilhelmsson, Coherent Non-Linear Interaction of Waves in Plasmas (Pergamon, Oxford, 1977).

[2] H. Goldstein, Classical Mechanics (Addison-Wesley, Reading, MA, 1980).

[3] P.A. Robinson and P. Drysdale, Phys. Rev. Lett. 77, 2698 (1996).

[4] G.I. de Oliveira, L.P.L. de Oliveira, and F.B. Rizzato, Physica D 104, 119 (1997).

[5] S.G. Thornhill and D. ter Haar, Phys. Rep. 43, 43 (1978).
[6] S.E. Gibson, D.L. Newman, and M.V. Goldman, Phys. Rev. E 52, 558 (1995).

[7] B. Malomed, D. Anderson, M. Lisak, and M.L. QuirogaTeixeiro, Phys. Rev. E 55, 962 (1997).

[8] P. Shukla, N.N. Rao, M.Y. Yu, and N.L. Tsintsadze, Phys. Lett. 138B, 1 (1986).

[9] R. Pakter and G. Corso, Phys. Plasmas 2, 4312 (1995).

[10] A. Lichtenberg and M.A. Lieberman, Regular and Stochastic Dynamics (Springer, New York, 1991). 